\title{
[ 384$]$
}

\section{III.}

TRALLES SENKWAGE,

und deren Gebrauch $\approx u m$ Abwägen aller Arcen von Körpern und $\approx u$ andern Versuchen.

1. Kurze Befchreibung diejer Senkwage,

$$
\text { Proferfor TRATLE, }
$$

Mitgliede der Akademie der Wiffenfchaften zu Berlin.

(Aus einen Briefe an den Profejfor Gilbert in Halle.)

- Um Ihrem Wunfche zu entiprechen, gebe Berlin den i6un Sept. 1908. ich linen hier eine kurze Befchreibung meiner hydroftatifchen Wage oder Senkwage.

Von einem hohlen Körper $A$, ('Taf. V, Fig. I ,) geht ein an demfelben befeftigter kurzer und dün. ner cylindrifcher Hals $B$ hervor, welcher mit einem dreifach gebogenen Arme $C, D, E$ vereinigt ift. Nan bringt den hohlen Körper in ein cylindrifches Glas, welches den Arm aufserhalb läfst, und fetzt das Glas auf ein dazu geeignetes Geftell fo hoch, dafs eine Schale $F$, die aĩ den unter dem Glafe befindlichen Theil des Arms angehängt wird, noch etwas Spielraum behält, fich auf und ab zu bewegen. Man giefst dann eine Flüligkeit, z. B. Waf- 


\section{[ 385 ]}

fer, in das Glas, und legt auf die Schale fo viel Gewicht, bis eive an dem dünnen Halle $E$ bemerkte Stelle gerade in die Oberfäche des Flalígen fallt. Die Wage fchwimmt alsdann; damit Ge fith aber gerade aufrecht erhaite, mufs der untere Theil des Arms $E F$ gehörig geftellt fegn, welches fich durch einiges Verfuchen leicht bewerkftelligen lätst. Man biegt zu dem Ende diefen Theil $E$ des Arms fo feitwärts, und verfchiebt die auf demfel'sen am Haken hin und her bewegliche Schale mit den ejngelegten Gewichten fo lange, bis man eine Stellung trifft, in welcher der Hals $B$ einen rechtwinkligen Stand mit der Oberfäche des Walfers oiler der FlüfGgkeit annimint. Diefe Stelle bemerist man auf $E$ ein fur alle Mainl.

Will man nun das Oewicht eines $\mathrm{B}$ örpers finden, fo legt man denfelben auf die Schale, und nimmt dafur Getvichts von derfelben weg, fo lange, bis die Waye genau bis an denfelben an dem Halle befindlichen Punkt eingetaucht ift, als fie es war, da die Schale blofs das Gewichtsfyftam enthielt. Das abgenommene Gewicht ift offenhar das Gewicht des Körpers. Es ift nur darauf zu achten, dafs die Temperatur der Flüfigkeit während der Operation fich nicht geändert habe. Ift man deffen nicht ficher, fo legt man das Gewichtsfyltem wiederum vollftāndig ftatt des Körpers auf, und Geht 24 , ob mit demfelben die Wage genau denfeiben Stand wieder annimmt, wie zuvor. Wenn dies nicht gofchieht, fo kann man fur das, was man mehr oder Anual. d. Plygfik. B. 30. St. 4. J. 1808. St. z3. C. c 


\section{[ $\left.\begin{array}{lll}386 & \end{array}\right]$}

weniger auflegen muls, in Beziehung auf das Gewicht des Kürpers die gehürige Berichtigung macisen.

So wie ich hier die Wage befobrieben babe, ift fie in ihrer einfachften Form. Eine hohle Glaskugel und ein meiallifcher - Draht, den man in ihre Oeffuung eirkittet und gehürig biegt, find alles, was man be:arf, um fich auf der Stelle eine Wage zu verfertigen, mit welcher man Körper, ein Paar Unzen lchwer, mit der Genanigkeit von einem funfzigften Theile eines Grans abıägen kann.

Das Princip, auf welches fe gegründet ift, unterfcheilet fie von den bisherigen Aräometern. Und es ift fondertar, dals man den Gellanken nicht fchon feit dem Gebrauche diefer Inftrumente zu bydroftatifchen Abwägungen geliabt hat, den Schwer. punkt mit Nutzen unter len Mittelpunlst des Drucks z.u bringen. Ish bin vor $18 \mathrm{~J}$ ahren auf diefe Einrichtung gerathen, da ich uachfuchte, wie ich mir eine hüchlt genauc Wage verfertigen Jaffen könnte, hequem auf Bergreifen zu gebrauchen. Eine Wage mittlerer Grüfse, welche niir der Mle:

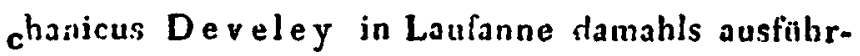
te, befitze ich roch. Stalt des einfachen gebogenen Arms find deren drei, welche aus einem geineinfclsaftlichen kiörper, (der den Hals $B$ aufnimmt,) oben ausgehen, und foch unten vermittelft eines Stucks in Geftalt eines $Y$, wo die 3 Linien Winkel vos $120 \mathrm{Grad}$ machen, vereinigen. Es ift eine Vorrichtung angebracht, durch welche der Punkt. 


\section{[ 387 ]}

an dem die Schale hängt, beiveglich ift, fo dafs man genau derr Schwerpankt der ganzen Malchise und den Mittelpunkt des Drurks in eine der Achfe derfelben urod dem Hallo pa:allele linie bringen kann. Da ich, wegen der Niatzlichkeit diefer Wage, die Ei:ırichtungen derfelhen theils viel:nalils in offentlichen Vorlefungen erklärt, theils Grlefirten unil Künftlern mit;ighlscist haise, fo ift fie, aucin ohne dals ich etwas tỉer fie hälte drucken lafien, bekannt genug gewurden. Nur foineinen nicht alle diefe Einrichtung der Wage be!salten zu haben, von wem Ge herrülirt; oiler auch mag es begegnet feyn, dals man geglaubt hat, diefe ldee als eine anfpruchsfreie Sarbe in ruhigen Befitz nehmen und erbalten zu künnen. In Thaer's Zeitf:hrift über Ackersoul u. Co w. hat unan mir felbft einen Holzfchnitt derfelben gewiefen, welcher der Figur ähnlich genug ilt, die ich mic der Feter in einem Briefe an den Hofrath Lich. tenberg in Güttingen beigezeichnet hatte. Die Wage wird auch, wis ich vernommen hahe, in Göttingen verfertigt. In Paris maclit fie lortin, dem ich fie ein Mlabl in feiner Werkftätte auf das Papier gebracht habe.

Uin das Jahe 1795 oder 1796 war irh heaufiragt, für die Anlage einer grolsen Wage, beftimmt, Laftwagen darauf zu wägen, ein Gutachten auszuarbei= ten. In diefem Gutachten habe ich gezeigt, difs diefe Einrichtung fich zu einem folchen $Z$ wecke feltr gut anordnen lalle. Jedoch habe ich fie dazu niciat 


\section{[ 388 ]}

befonders dringend anempfot:len, weil ich die is England gebräuchliche Einrichtung, ob fee gleich koltbarer ift, doch filr zweckmälsiger halie, wegen der Perfonen, denen man den Gebrauch ühurJaffen mufs. Für Verfuche bediene ich mich aber nur in ganz befondern Fällen der eigentlich liati. fchen Wage, indem meine hyitroftatifche Senkivage eine Genauigkeit und Sicherheit giebt, welche man felten bei jenen findet. Ich hielt es daher far nützlich, der ausfubrlichen Befchreibung und der mathematifclien Theorie derfelben eine befondere Ablhandlung $z u$ widmen, welclie ich vor vier Jahren hier in der Akademie der Wiffenfchaften vorgo. lefen habe. Un hier nicht voreilig das zu wiederhohlea, was dein Publicuin nàber bekannt werden wird, went die Abhandlungen der Akarlemie für jenes Jahr erfcheinen, habe jch mich nur fehr kurz gefalst, doch wird jeder Phyfiker in Stande feyn, fich nach dem Wenigen eine lolche Vorrichtung felbft anzuordnen, oder von jedem Künftler verfertigen zu laifen. Ich bemerke nur beiläufig noch, dafs fie fich gut gebrauchen lälst, um magnetifche und electrifche Wirkungen zu beobachten, wenn diefe Wirkungen eine drehende Bewegung hervorbringen, 2. B. wenn man einen Magnetftab auf die Schale legt, u. f. w. 